

\title{
Bilateral oligopoly and quantity competition
}

\author{
Alex Dickson \\ Roger Hartley
}

June 2009

Economics

School of Social Sciences

The University of Manchester

Manchester M13 9PL 


\title{
Bilateral oligopoly and quantity competition
}

\author{
Alex Dickson*and Roger Hartley ${ }^{\dagger}$
}

June 22, 2009

\begin{abstract}
Bilateral oligopoly is a strategic market game with two commodities, allowing strategic behavior on both sides of the market. When the number of buyers is large, such a game approximates a game of quantity competition played by sellers. We present examples which show that this is not typically a Cournot game. Rather, we introduce an alternative game of quantity competition (the market share game), which yields the same equilibria as the many-buyer limit of bilateral oligopoly, under standard assumptions on costs and preferences. We also show that the market share and Cournot games have the same equilibria if and only if the price elasticity of the latter is one. These results lead to necessary and sufficient conditions for the Cournot game to be a good approximation to bilateral oligopoly with many buyers and to an ordering of total output when they are not satisfied.
\end{abstract}

\section{Introduction}

In this paper we investigate quantity competition in a market with few sellers and many buyers, but in which price-taking behavior is not imposed on the latter by fiat. We model the interactions this gives rise to as a game of bilateral oligopoly. This is a strategic market game with two commodities: a consumption good as well as commodity money, in which both buyers and sellers are assumed to act strategically. When the set of buyers becomes large, we might expect their individual choices to approach price-taking behavior and, if the number of sellers remains small, this will lead to a game of quantity competition. The question then arises: what is the nature of this quantity competition?

We show by example that, whilst there are types of buyer preferences for which the answer to this question is the Cournot game, this will not be

\footnotetext{
${ }^{*}$ Corresponding author; Department of Economics, University of Strathclyde, Sir William Duncan Building, 130 Rottenrow, Glasgow, G4 0GE, email: alex.dickson@strath.ac.uk, tel: +44 (0)141548 3849.

${ }^{\dagger}$ University of Manchester, Oxford Road, Manchester, M13 9PL, UK, email: roger.hartley@manchester.ac.uk, tel: +44 (0)161 2754799.
} 
true in general. This raises additional questions: under what circumstances is Cournot equilibrium a good approximation to bilateral oligopoly with many buyers and, when it is not, what is the relationship between Cournot equilibrium and the many-buyer limit of equilibria in bilateral oligopoly?

At the heart of the answers we offer to these questions is a model of quantity competition which is a variant of the Cournot game. The latter implicitly assumes the existence of a mechanism, which we label an auctioneer, to ensure markets clear once sellers have committed to their quantity decisions and it will prove convenient to model this as a two-stage game in which sellers choose quantities in the first stage and the auctioneer, viewed as a player, moves in the second stage. We suppose that the auctioneer chooses aggregate revenue $R$ and has a payoff function which, given aggregate output of sellers $X$, is maximized when the revenue raised at the market-clearing price when output is $X$ is exactly equal to $R$. The first stage of this sequential game is a conventional Cournot game and its subgame perfect equilibrium is output-equivalent to the Cournot equilibrium. Our alternative model is derived from this game by reversing the timing of auctioneer and sellers.

This yields a market sharing game in which the auctioneer chooses revenue at the first stage and sellers choose quantities in the second; strategy spaces and payoffs are unchanged. We show that, if sellers' costs are increasing and convex and demand is decreasing and has a choke-off price that is not too low, the market share game will have a unique non-autarkic equilibrium. (We also consider the possibility that the auctioneer plays simultaneously with the sellers in a single stage game and show that the outputs of sellers in the Nash equilibrium of this game coincide with those on the equilibrium path of the market share game.) When the set of buyers is made large by replicating a finite set of types whilst proportionately reducing the weight of each buyer, bilateral oligopoly can be recast in a form that resembles the market share game. This observation can be used to show that outcomes in the non-autarkic equilibrium of bilateral oligopoly approach those of the market share game. Furthermore, buyers behave as price takers facing the equilibrium strategic price.

We can use these observations to investigate the relationship between the many-buyer limit of bilateral oligopoly and the Cournot game, since the former approaches the market share game. Whilst markets clear in both the market share and Cournot games, the choices of sellers on the equilibrium path of the subgame perfect equilibria under the two timing structures may differ. If the Cournot game has an equilibrium at which the elasticity of demand is unity, then the price and profile of outputs are shown to be exactly the same in this equilibrium as in the non-autarkic equilibrium of the market share game. Under this supposition, the manybuyer limit of non-autarkic equilibria in bilateral oligopoly agrees in price and outputs with Cournot oligopoly. However, if the Cournot game has 
an equilibrium at which demand elasticity exceeds one, aggregate output in the latter equilibrium will exceed the aggregate output in the market share game. When the elasticity is less than one, this ordering is reversed.

General strategic market games were originally introduced by Shapley and Shubik (see, for example, [14]), and subsequently studied extensively (see Dubey [7] or Giraud [10] for comprehensive reviews). The model we use is a specialization of these multiple-commodity frameworks to a market for a single consumption commodity with trade in a commodity money, first studied by Gabszewicz and Michel [8]. The relationship between equilibria in strategic market games and Walrasian equilibria with many agents on both sides of the market has seen much attention and is well understood (see Mas-Colell [11] for a review). However, the relationship between bilateral oligopoly with many buyers but few sellers and Cournot competition (or more generally between strategic market games and general equilibrium models of Cournot competition discussed by Gabszewicz and Vial [9]) is less well explored. There are few recent exceptions to this: Codognato [2] provides two examples demonstrating convergence in one case, and non-convergence in the other, of outcomes in bilateral oligopoly to Cournot outcomes as the number of buyers increases by replication. Busetto, Codognato and Ghosal [1] also highlight this non-equivalence in a model with a continuum of agents with some atoms (the oligopolists), and proceed by considering the relationship between a two-stage respecification of the strategic market game and Cournot's model. We contribute to this literature by identifying a game that is the many-buyer limit of bilateral oligopoly and develop necessary and sufficient conditions under which outcomes in this model of quantity competition will be equal to those in Cournot oligopoly.

Our analysis of quantity competition and comparison between models is made possible by exploiting the aggregative properties of the games we consider. 'Aggregativeness' in games was originally recognized and exploited by Selten [13]. The method was employed by Novshek [12] in proving existence of equilibrium in Cournot competition, and it has been successfully used in the study of contests (see, for example, Cornes and Hartley [3], Stein [15] and Szidarovsky and Okuguchi [16]), the results from which we apply directly in some of our analysis. Dickson and Hartley [6] extended the ideas behind the analysis of these games with a single aggregate to study bilateral oligopoly using an extension of the methodology. The current paper builds on this by comprehensively investigating the nature of bilateral oligopoly when the number of traders on only one side of the market becomes large.

The rest of the paper is structured as follows. In the next section we present the model of bilateral oligopoly with replicated buyer types and analyze the many-buyer limit in two examples. In one, equilibria approach the Cournot equilibrium, but in the other this is typically not the case. In Section 3, we formulate the market share game and present conditions under which it has a unique non-autarkic equilibrium. In Section 4, we 
investigate conditions under which the many-buyer limit of equilibria in bilateral oligopoly is the same as that of the market share game. Combining these results in Section 5, we show that unit elasticity of demand at the Cournot equilibrium is necessary and sufficient for this equilibrium to be a good approximation to that of bilateral oligopoly with many players. We also study the ordering of aggregate output in Cournot and (limit) bilateral oligopoly games when equilibrium elasticity differs from one. Section 6 concludes and some proofs omitted from the main exposition are collected in an appendix.

\section{Bilateral Oligopoly}

In this section, we outline a variant of the model of bilateral oligopoly introduced by Gabszewicz and Michel [8]. This model, which is a specialized strategic market game in the Shapley-Shubik vein [14], permits strategic behavior on each side of a market in which signals are quantity-based.

There is one consumption commodity, as well as commodity money, and two sets of agents: sellers and buyers. Since we will be interested in equilibria of games with many buyers, we consider a sequence of economies, which we denote $\mathcal{E}^{m}$ for $m=1,2,3, \ldots$. All economies share the same set of sellers: a set $I^{\mathrm{S}}=\{1, \ldots, n\}$, with at least two members. The set of buyers is formed by replication of a finite, non-empty set of types $\mathcal{I}^{\mathrm{B}}$. In $\mathcal{E}^{m}$, there are $m$ replicas of each buyer, so the buyer set is $I^{\mathrm{B} m}=\mathcal{I}^{\mathrm{B}} \times\{1, \ldots, m\}$. To avoid limiting equilibrium prices becoming infinite, we will give each buyer a weight of $1 / m$ (and sellers unit weight). If $\mathcal{I}^{\mathrm{B}}$ is a singleton, we consider only values of $m$ exceeding 2 .

We suppose that each seller $i$ is endowed only with a production technology that can supply $x(\geq 0)$ units of the consumption good at a cost $C_{i}(x)$, denominated in terms of commodity money. A buyer of type $i$ is endowed only with $e_{i}>0$ units of money and can place a money bid $b \in\left[0, e_{i}\right]$. The market aggregates supply to $X=\sum_{j \in I^{\mathrm{S}}} x_{j}$ and money bids to $B=\sum_{j \in I^{\mathrm{B} m}} b_{j} / m$ and determines the strategic (market clearing) price as $p=B / X$ (so long as ${ }^{1} B, X>0$ ). A seller who supplies $x$ receives revenue $x p$ and evaluates supply choices according to the payoff function:

$$
x p-C_{i}(x)=\frac{x}{X} B-C_{i}(x) .
$$

Note that seller $i$ receives a share of total revenue proportional to its supply; a fact we exploit in the sequel. Similarly, buyers receive a share of total output of the good proportional to their bid. After trading a typical buyer has $b / p$ units of the good and is left with $e_{i}-b$ units of money. We assume

\footnotetext{
${ }^{1}$ If either $B$ or $X$ are zero, the market is deemed closed and no trade takes place.
} 
the preferences of buyers are representable by a utility function, $u_{i}$, so type $i \in \mathcal{I}^{\mathrm{B}}$ receives payoff

$$
u_{i}\left(\frac{b}{B} X, e_{i}-b\right)
$$

We study pure-strategy Nash equilibria of these games and observe that there is always a no-trade equilibrium in which $x_{i}=0$ for all $i \in I^{\mathrm{S}}$ and $b_{i}=0$ for all $i \in \mathcal{I}^{\mathrm{B}}$. We therefore focus on non-autarkic equilibria ${ }^{2}$ in which $B, X>0$ and, in particular, study the limit of these equilibria as $m \rightarrow \infty$. It is instructive to compare these limits with Cournot equilibria in which competitive demand is the aggregate demand of buyers in $\mathcal{I}^{\mathrm{B}}$, acting as price-takers. We commence with two examples; the first presents an adaptation of a result of Codognato [2] to our framework.

Example 1 Suppose there are two sellers with identical quadratic costs given by $\frac{1}{2} x^{2}+\gamma x$, where $\gamma \geq 0,{ }^{3}$ and one buyer type with a unit endowment of money and preferences given by $u\left(y_{1}, y_{2}\right)=y_{1}^{\alpha} y_{2}^{1-\alpha}$, where $0<\alpha<1$. Competitive inverse demand is $p(X)=\alpha / X$ and there is a unique ${ }^{4}$ Cournot equilibrium in which aggregate supply is

$$
\tilde{X}^{\mathrm{C}}=-\gamma+\sqrt{\gamma^{2}+\alpha} .
$$

In bilateral oligopoly the first-order conditions can be used to verify that, for $m \geq 2$, there is a non-autarkic equilibrium of $\mathcal{E}^{m}$ in which the weighted aggregate bid is

$$
\tilde{B}^{m}=\frac{\alpha m-\alpha}{m-\alpha}
$$

and the aggregate offer is

$$
\tilde{X}^{m}=-\gamma+\sqrt{\gamma^{2}+\tilde{B}^{m}} .
$$

A result in [6] (Theorem 5.3) can be used to show that this is the unique non-autarkic equilibrium of $\mathcal{E}^{m}$.

As $m \rightarrow \infty, \tilde{B}^{m} \rightarrow \alpha$ and $\tilde{X}^{m} \rightarrow \tilde{X}^{\mathrm{C}}$. It can also be checked that $\tilde{B}^{m} / \tilde{X}^{m}$ converges to the market price in the Cournot equilibrium and buyers' equilibrium consumption approaches price-taking behavior at this price.

\footnotetext{
${ }^{2}$ Under the assumptions on cost functions and preferences we introduce later, there is a unique non-autarkic equilibrium in which buyers of the same type make the same bids. We therefore loosely refer to behavior of types of buyers, meaning the common choice of all buyers of that type.

${ }^{3}$ The results in this (and the next) example continue to hold for $\gamma<0$ provided we impose that marginal costs are positive along the best response functions. We omit details of the parameter restrictions that guarantee this.

${ }^{4}$ This example satisfies the uniqueness conditions of Szidarovsky and Yakowitz [17].
} 
In this example, market outcomes in bilateral oligopoly approach the outcome at the Cournot equilibrium in the many-buyer limit. Indeed, it is straightforward to verify that this extends to any set of buyer types with Cobb-Douglas preferences and that symmetry amongst sellers is inessential. However, the next example shows that Cobb-Douglas preferences are working particularly hard to give us this conclusion.

Example 2 Suppose there are two sellers with identical quadratic costs given by $\frac{1}{2} x^{2}+\gamma x$, where $\gamma \geq 0$, and one buyer type with a unit endowment of money and quadratic preferences given by $u\left(y_{1}, y_{2}\right)=\beta y_{1}-\frac{1}{2} y_{1}^{2}+y_{2}$, where $\beta>\gamma$. In this case, competitive inverse demand is $p(X)=\max \{\beta-X, 0\}$ and equilibrium supply at the unique ${ }^{5}$ Cournot equilibrium is

$$
\tilde{X}^{\mathrm{C}}=\frac{\beta-\gamma}{2} .
$$

In bilateral oligopoly, the uniqueness theorem in [6] can be applied again to conclude that there is a unique non-autarkic equilibrium of $\mathcal{E}^{m}$ for all large enough $m$, in which the aggregate offer is

$$
\tilde{X}^{m}=\frac{(m-1) \beta-2 \gamma m}{2 m-1}
$$

and the aggregate bid is

$$
\tilde{B}^{m}=\frac{m-1}{m} \tilde{X}^{m}\left(\beta-\tilde{X}^{m}\right) .
$$

Note that

$$
\lim _{m \rightarrow \infty} \tilde{X}^{m}=\frac{\beta-2 \gamma}{2} .
$$

and this limit differs from $\tilde{X}^{\mathrm{C}}$ except when $\gamma=0$. A fruitful way of displaying this observation is to note that the price elasticity of competitive demand at the Cournot equilibrium, $\eta\left(\tilde{X}^{C}\right)$, satisfies

$$
\operatorname{sign}\left\{\eta\left(\tilde{X}^{\mathrm{C}}\right)-1\right\}=\operatorname{sign} \gamma \text {. }
$$

It follows that the limiting aggregate offer is equal to $\tilde{X}^{C}$ if and only if $\eta\left(\tilde{X}^{\mathrm{C}}\right)=1$. If this holds, we can also verify convergence of buyers' consumption and the market price to Cournot equilibrium values. Otherwise, $\tilde{X}^{\mathrm{C}}>(<) \lim _{m \rightarrow \infty} \tilde{X}^{m}$ if and only if $\eta\left(\tilde{X}^{\mathrm{C}}\right)>(<) 1$.

\footnotetext{
${ }^{5}$ Whilst this example does not satisfy the conditions of Szidarovsky and Yakowitz [17], weaker conditions that encompass our example are shown in section 4.2 of Vives [18] to give rise to a unique equilibrium.
} 
In the second example, convergence to Cournot equilibrium is exceptional and this suggests that there may be an alternative quantity competition game played by sellers that better represents bilateral oligopoly with many buyers. In the next two sections, we describe and analyze such a game (the market share game) and then discuss the relationship between its equilibrium and the limits of the equilibria of the sequence of bilateral oligopoly games.

\section{Market share game}

In this section, we introduce a quantity-competition game played by profitmaximizing sellers in which buyers are assumed to be price takers. It will be convenient to view this game as a variation of Cournot oligopoly and we start by casting the latter as a two-stage game, denoted $\mathcal{G}^{+}$. In the first stage of $\mathcal{G}^{+}$, each seller $i \in I^{\mathrm{S}}$ chooses output $x_{i}$ and this determines total output $X=\sum_{j \in I^{\mathrm{S}}} x_{j}$. In the second stage, an auctioneer sets a price to clear the market. Note that setting a price in this game is equivalent to choosing the total revenue received by sellers, which write $R$. The revenue of seller $i$ is equal to a share $x_{i} / X$ of $R$. We can view the auctioneer as a player whose payoff is maximized if and only if $R$ is equal to the revenue received from buyers when price is chosen so that aggregate demand is $X$ (i.e. such that the market clears).

The market share game, denoted $\mathcal{G}^{-}$, is obtained simply by changing the order of the stages so that the auctioneer chooses $R$ in the first stage. In the second stage, sellers compete for a share of this revenue by choosing outputs simultaneously. Formally, the second stage game takes the form of a Tullock contest with, in general, non-linear costs. We also discuss the single-stage game $\mathcal{G}^{0}$ in which the auctioneer moves at the same time as the sellers.

Seller $i \in I^{\mathrm{S}}$ in all three games has strategy set $\left\{x_{i}: x_{i} \geq 0\right\}$ and payoff equal to its profit function $\pi_{i}$, where

$$
\pi_{i}\left(x_{1}, \ldots, x_{n}\right)=\frac{x_{i}}{X} R-C_{i}\left(x_{i}\right)
$$

if $x_{i} \neq 0$ and $\pi_{i}=0$ if $x_{i}=0 . C_{i}$, the cost function of seller $i$, is assumed to be smooth, increasing and exhibit non-increasing returns.

Assumption A1 For all $i \in I^{\mathrm{S}}$, the cost function $C_{i}$ is convex, continuously differentiable and satisfies $C_{i}(0)=0$ and $C_{i}^{\prime}\left(x_{i}\right) \geq 0$ for $x_{i} \geq 0$ with strict inequality if $x_{i}>0$.

Where appropriate, we interpret $C_{i}^{\prime}(0)$ as a one-sided derivative.

Passive buyer behavior is captured by the inverse demand function $p$, which is decreasing where positive, approaches or is equal to 0 for large $X$ and does not vanish. 
Assumption A2 Inverse demand $p$, defined for $X>0$ is continuous, strictly decreasing where positive and satisfies

$$
\inf _{X>0} p(X)=0<\sup _{X>0} p(X) .
$$

The auctioneer has strategy set $\{R: R \geq 0\}$ and payoff $\xi(X, R)$, where

$$
\xi(X, R) \begin{cases}=0 & \text { if } X>0 \text { and } R=X p^{+}(X), \\ =0 & \text { if } X=0 \text { and } R=0, \text { and } \\ <0 & \text { otherwise. }\end{cases}
$$

In $\mathcal{G}^{+}$, we seek a subgame perfect equilibrium and note that, in any second-stage subgame of $\mathcal{G}^{+}$in which the aggregate output in the first stage is $X$, the auctioneer maximizes payoff by choosing $R=X p(X)$. This means that the payoff of seller $i$ in the first stage is $x_{i} p(X)-C_{i}\left(x_{i}\right)$ and shows that the strategy profile of sellers in a subgame perfect equilibrium is a Cournot equilibrium.

In $\mathcal{G}^{-}$, there is always an autarkic subgame perfect equilibrium in which the auctioneer plays $R=0$, for then every seller chooses zero output in the subgame determined by $R=0$ and the auctioneer receives maximum (zero) payoff. We therefore seek non-autarkic subgame perfect equilibria in which $R>0$ (and $X>0$ on the equilibrium path). The simultaneous-move game $\mathcal{G}^{0}$ also has an autarkic equilibrium, so again we study non-autarkic Nash equilibria in which the auctioneer plays $R>0$.

In the next two subsections, we present an additional condition which ensures that $\mathcal{G}^{-}$has a non-autarkic subgame perfect equilibrium and that such an equilibrium must be unique.

\subsection{Backwards induction: Stage 2}

We will find it convenient to use $\mathcal{G}(R)$ to denote the second-stage subgame when the auctioneer plays $R$ in the first stage. Note that payoffs are given by (2), so that, if $R>0$, this is a rent-seeking contest with proportional contest success function ${ }^{6}$, rent $R$ and cost functions $C_{i}$. We can exploit the aggregative nature of such games ${ }^{7}$ in analyzing their equilibria. Rather than study fixed points of ( $n$-dimensional) best response maps directly, we use the techniques described in [4].

In that paper, it was shown that, for any seller $i$ and any $X>0$, there is a unique strategy $x_{i} \geq 0$ such that seller $i$ plays $x_{i}$ in any Nash equilibrium of $\mathcal{G}(R)$ in which the sum of strategies is $X$. For any $i$ this allows us to

\footnotetext{
${ }^{6}$ With an appropriate change of variables if necessary, $\mathcal{G}(R)$ is strategically equivalent to a generalized Tullock contest with unit average cost and logistic contest success function [3].

${ }^{7}$ The payoff of player $i$ is a function only of $x_{i}$ and $X$.
} 
define a share function which maps any $X>0$ to $\sigma_{i}=x_{i} / X$. We write $s_{i}(X ; R)$ for the value of this share function and note that $s_{i}(X ; R)=\sigma_{i}$ if and only if $\sigma_{i} X$ is a best response by seller $i$ to any strategy profile of the other sellers which satisfies $\sum_{j \neq i} x_{j}=\left(1-\sigma_{i}\right) X$. Since $\pi_{i}$ is a concave function of $x_{i}$, the first-order conditions are necessary and sufficient for $x_{i}$ to be a best response in $\mathcal{G}(R)$. These conditions imply that $s_{i}(X ; R)=\sigma_{i}$ if and only if

$$
L^{\mathrm{M}}\left(\sigma_{i}, X ; R\right)=\left(1-\sigma_{i}\right) \frac{R}{X} \leq C_{i}^{\prime}\left(\sigma_{i} X\right),
$$

with strict inequality if $\sigma_{i}>0$.

The construction of the value of $s_{i}$ for given $X$ and $R$ is illustrated in Figure 1. In this figure, $L^{\mathrm{M}}$ and $C_{i}^{\prime}$, considered as functions of $\sigma_{i}$, are drawn as solid lines. Since $L^{\mathrm{M}}$ is strictly decreasing and $C_{i}^{\prime}$ non-decreasing in $\sigma_{i}$ the lines can cross at most once. If they do not cross, the $L^{\mathrm{M}}$ line must lie below the $C_{i}^{\prime}$ line, in which case the first order conditions imply $s_{i}(X ; R)=0$. This shows that the share function is well-defined for all $X, R>0$. Furthermore, an increase in $X$ pivots $L^{\mathrm{M}}$ down and $C_{i}^{\prime}$ up (under Assumption A1), from which we can deduce that the share function is decreasing in $X$ where positive. Figure 1 also makes the remaining assertions in the following lemma plausible. For a formal proof see [3].



Figure 1: Share functions in the market share game for $R$ and $R^{\prime}>R$. 
Lemma 3.1 Suppose Assumption $\boldsymbol{A} \mathbf{1}$ is satisfied and $i \in I^{\mathrm{S}}$. For any $R>0$, there is a continuous share function $s_{i}(X ; R): \mathbb{R}_{++}^{2} \rightarrow[0,1]$, where $s_{i}$

1. is strictly decreasing where positive,

2. approaches unity as $X \rightarrow 0$, and

3. is eventually equal to zero or approaches zero as $X \rightarrow \infty$.

Share functions allow us to study equilibria using the observation that there is an equilibrium in $\mathcal{G}(R)$ with aggregate supply $X$ if and only if

$$
S(X ; R)=\sum_{j \in I^{\mathrm{S}}} s_{j}(X ; R)=1,
$$

in which case the equilibrium strategy profile is $\left(X s_{1}(X ; R), \ldots, X s_{n}(X ; R)\right)$. It follows from the lemma that $S(X ; R)$ is continuous, exceeds unity for small enough $X$, approaches or is equal to zero for large enough $X$ and is strictly decreasing where positive. This implies that (5) holds for exactly one $X$ and this entails a unique equilibrium.

Lemma 3.2 If Assumption $\boldsymbol{A} 1$ is satisfied, then $\mathcal{G}(R)$ has a unique equilibrium for any $R>0$.

We write $\mathcal{X}(R)$ for the equilibrium value of $X$ in $\mathcal{G}(R)$. To study equilibria of $\mathcal{G}^{-}$, we will need to analyze how $\mathcal{X}(R)$ varies with $R$. Firstly, we observe that $\mathcal{X}(R)$ is continuous in $R>0$. This follows from a compactness argument, using the fact that $S(\mathcal{X}(R) ; R)=1$ along with properties of $S$ inherited from those of individual share functions set out in Lemma 3.1. Furthermore, $L^{\mathrm{M}}$ is strictly increasing in $R$ and decreasing in $\sigma_{i}$, whereas $C_{i}^{\prime}$ is constant in $R$ and Assumption $\mathbf{A} \mathbf{1}$ implies that it is non-decreasing in $\sigma_{i}$. It follows that $s_{i}(X ; R)$ increases with $R$ for fixed $X$, strictly if $s_{i} \in(0,1)$. This is illustrated in Figure 1 , in which we display $L^{\mathrm{M}}$ when $R$ is increased to $R^{\prime}$ as a dashed line. We deduce that, for any $X>0$, the aggregate share function $S(X ; R)$ increases with $R$ where it is positive. Since $S(\mathcal{X}(R) ; R)=1$, we may use Lemma 3.1 to conclude that $\mathcal{X}(R)$ increases with $R$. In the appendix, we extend this result to show that this increase is no faster than $R$ and that $\mathcal{X}(R)$ is not bounded above. These observations are summarized in the following proposition ${ }^{8}$.

Proposition 3.3 If Assumption $\boldsymbol{A} 1$ is satisfied, $\mathcal{X}(R)$ is continuous in $R>0$, unbounded above, strictly increasing and the dissipation ratio $\rho(R)=$ $\mathcal{X}(R) / R$ is non-increasing in $R>0$.

\footnotetext{
${ }^{8}$ We believe that the observation that the dissipation ratio is decreasing in the rent is a new result.
} 


\subsection{Backwards induction: Stage 1}

Having characterized the equilibrium in second-stage subgames, we now turn to the first stage, in which the auctioneer chooses $R$ to maximize $\xi(X, R)$, where the equilibrium in $\mathcal{G}(R)$ implies that $X=\mathcal{X}(R)$. Since $\mathcal{X}(0)=0$ and $\xi \leq 0$, the auctioneer can always maximize payoff by choosing $R=0$. This leads to the autarkic equilibrium of $\mathcal{G}^{-}$in which all sellers produce zero output. However, if there is also an $R>0$ satisfying $R=f(\mathcal{X}(R))$, where $f(X)$ is the revenue function: $f(X)=X p(X)$, this too will achieve the maximum and $\mathcal{G}^{-}$will have a non-autarkic subgame perfect equilibrium. We can use Proposition 3.3 to derive the conditions under which there is such an $R$ and show that it is unique. This leads to the following theorem characterizing equilibria of $\mathcal{G}^{-}$. The proof is in the appendix.

Theorem 3.4 If Assumptions $\boldsymbol{A} 1$ and $\boldsymbol{A} 2$ are satisfied and $\mathcal{G}^{-}$has a nonautarkic subgame perfect equilibrium this equilibrium is unique and the auctioneer chooses $\tilde{R}=f(\tilde{X})$, where $\tilde{X}=\mathcal{X}(\tilde{R})$. Such an equilibrium exists if and only if

$$
\sum_{j \in I^{\mathrm{S}}}\left[1-\frac{C_{j}^{\prime}(0)}{p(0)}\right]>1 .
$$

If the inequality in the theorem fails, there is only the autarkic equilibrium. Note that (6) is satisfied if $p(0)$ is infinite (for example, isoelastic demand) irrespective of sellers' cost functions. This is also true if marginal cost falls to zero at zero output for at least one seller. However, the theorem does not imply that profitable trade is always realized. If minimal marginal cost is less than the choke-off price for some sellers: $C_{i}^{\prime}(0)<p(0)$, production is profitable for such sellers. But, this does not imply that (6) is necessarily satisfied: strategic factors may prevent gains from trade from being exploited.

\subsection{Simultaneous moves}

Now consider the game $\mathcal{G}^{0}$ in which the auctioneer and sellers play simultaneously. In a Nash equilibrium of $\mathcal{G}^{0}$, seller $i$ chooses output $\hat{x}_{i}$ to maximize $\pi_{i}$, given by (2), where $R$ is the revenue chosen by the auctioneer. It is straightforward to confirm that $\hat{x}_{i}=s_{i}(\hat{X} ; R) \hat{X}$, where $\hat{X}=\sum_{j \in I^{\mathrm{S}}} \hat{x}_{j}$ and we can interpret $\hat{X}$ as a collective best response by sellers to the auctioneer's strategy. The auctioneer's best response to an aggregate output of $\hat{X}$ by sellers maximizes $\xi(\hat{X}, R)$ with respect to $R$. Thus, $\hat{X}=\mathcal{X}(\hat{R})$ where $\hat{R} \in \arg \max _{R} \xi(\hat{X}, R)$ and hence $\hat{R}=f(\mathcal{X}(\hat{R}))$. These conditions are equivalent to those determining outputs and revenue on the equilibrium path of $\mathcal{G}^{-}$. We conclude that $\mathcal{G}^{0}$ is outcome equivalent to $\mathcal{G}^{-}$in the sense that, 
in equilibrium, the strategies chosen by sellers in $\mathcal{G}^{0}$ are the same as those on the equilibrium path of $\mathcal{G}^{-}$. Theorem 3.4 has the following corollary.

Corollary 3.5 If Assumptions $\boldsymbol{A} 1$ and $\boldsymbol{A} 2$ are satisfied, $\mathcal{G}^{0}$ has a unique non-autarkic equilibrium if and only if (6) holds.

\section{The many-buyer limit of bilateral oligopoly}

In this section we consider the equilibria of bilateral oligopoly as $m \rightarrow \infty$. To illustrate our results, we first return to Example 2 and calculate the equilibrium of the market-share game.

Example 2 revisited. Payoffs in the second-stage subgame $\mathcal{G}(R)$ of a market-share game in which costs are as in Example 2 and the auctioneer chooses $R$ in the first stage are

$$
\frac{x}{X} R-\frac{1}{2} x^{2}-\gamma x .
$$

This means that $\mathcal{X}(R)$, the equilibrium value of $X$ in $\mathcal{G}(R)$, satisfies

$$
R=[\mathcal{X}(R)]^{2}+2 \gamma \mathcal{X}(R) .
$$

If preferences also agree with those in Example 2, inverse demand is $p(X)=$ $\beta-X$. Setting $\tilde{R}$ equal to $\mathcal{X}(\tilde{R}) p(\mathcal{X}(\tilde{R}))$ and solving for $\mathcal{X}(\tilde{R})$ gives

$$
\mathcal{X}(\tilde{R})=\frac{\beta-2 \gamma}{2}
$$

which is the equilibrium aggregate supply in the market-share game.

Comparing this with the original example, we observe that, as $m \rightarrow \infty$, the limit of the equilibrium aggregate output in $\mathcal{E}^{m}$ approaches $\mathcal{X}(\tilde{R})$, the equilibrium output in the market share game. This is a general result and to show this, we start by recalling from our discussion in Section 2 that, in bilateral oligopoly, sellers' supply decisions are aggregated to $X=$ $\sum_{j \in I^{\mathrm{S}}} x_{j}$ and buyers' bids are aggregated to $B=\sum_{j \in I^{\mathrm{B} m}} b_{j} / m$. The payoff to seller $i$ is then given by $\left(x_{i} / X\right) B-C_{i}\left(x_{i}\right)$, and the first-order conditions can be written (in terms of shares $\left.\sigma_{i}=x_{i} / X\right)$ as $L\left(\sigma_{i}, X ; B\right) \leq C_{i}^{\prime}\left(\sigma_{i} X\right)$ with equality if $\sigma_{i}>0$, where $L\left(\sigma_{i}, X ; B\right)=\left(1-\sigma_{i}\right) B / X$. Recalling the analysis of condition (4) in our discussion of the market share game, we can conclude that the aggregate supply of the sellers in a bilateral oligopoly equilibrium in which the aggregate bid of the buyers is $B>0$ will be $\mathcal{X}(B)$, with the properties presented in Proposition 3.3 when $R$ is replaced by $B$. This is the first similarity with the market share game, and indicates equivalence in seller behavior in bilateral oligopoly with that in subgames of 
the market share game. Next we go on to explore the buyers' behavior and show that, collectively, it mimics that of the auctioneer when the number of buyers is many.

In order to deduce this we appeal to a characterization of equilibrium in bilateral oligopoly from [6]. In particular, aggregate demand that is consistent with a Nash equilibrium in which the price is $p$ can be represented by a strategic analogue of the competitive demand function. Specifically, under binormality of buyers preferences ${ }^{9}$ it was shown that, for any $p>0$ there is a unique value of the aggregate bid of buyers in an equilibrium of any bilateral oligopoly game with buyer set $I^{\mathrm{B} m}$. The ratio of this aggregate bid to price defines the strategic demand function $\mathcal{D}^{m}(p)$ which can be shown to be continuous, strictly decreasing in $p$ and unbounded as $p \rightarrow 0$ [6, Lemma 5.1]. Therefore, $\mathcal{D}^{m}$ is invertible to a continuous decreasing function which we denote $p^{\mathrm{B} m}(X)$. If the aggregate supply from the sellers is $X$, the price that will emerge in the market given the buyers' optimal actions (taking into account all the strategic effects) is $p^{\mathrm{B} m}(X)$. It also follows from these observations that $p^{\mathrm{B} m}$ satisfies Assumption A2.

As buyers become more numerous, their individual market power diminishes and we might expect their behavior to approximate price taking. The following lemma, proved in the appendix, captures this at the level of aggregates.

Lemma 4.1 Suppose all buyers' preferences are binormal. Then as $m \rightarrow$ $\infty$

$$
p^{\mathrm{B} m}(X) \rightarrow p(X)
$$

pointwise. Moreover, on any compact subset of $\mathbb{R}_{++}$, this convergence is uniform.

It follows from the observations in the preceding paragraphs that, in an equilibrium of $\mathcal{E}^{m}$ in which the aggregate bid is $B$, the strategic price will be $p^{\mathrm{B} m}(\mathcal{X}(B))$. Since the price is constructed as the ratio of aggregate bid to aggregate supply, it is straightforward to see that there is a Nash equilibrium in bilateral oligopoly with aggregate bid $B>0$ if and only if ${ }^{10}$

$$
\frac{B}{\mathcal{X}(B)}=p^{\mathrm{Bm}}(\mathcal{X}(B)) \text {. }
$$

\footnotetext{
${ }^{9}$ Binormality of preferences requires that the marginal rate of substitution increases with moves to the north-west: if $\partial_{i}\left(y_{1}, y_{2}\right)$ represents the marginal rate of substitution, $y_{1} \leq y_{1}^{\prime}, y_{2} \geq y_{2}^{\prime} \Rightarrow \partial_{i}(y) \geq \partial_{i}\left(y^{\prime}\right)$ where the final inequality is strict if $y_{1}<y_{1}^{\prime}$ and $y_{2}>0$.

${ }^{10}$ In [6] it was shown that equilibria in bilateral oligopoly are in $1-1$ correspondence with intersections of the strategic demand curve with a similar strategic supply curve constructed for sellers. Equation (7) states the same result in a form more readily applicable in the current context.
} 
We may apply Theorem 3.4 to deduce that, provided

$$
\sum_{j \in I^{\mathrm{S}}}\left[1-C_{j}^{\prime}(0) / p^{\mathrm{B} m}(0)\right]>1
$$

there will be a unique $\tilde{B}^{m}>0$ which satisfies (7). It follows from Lemma 4.1 that, if (6) holds, there will be a positive integer $\bar{m}$ such that (8) is satisfied for all $m \geq \bar{m}$. Hence, $\tilde{B}^{m}$ is well-defined when buyers are sufficiently numerous: a non-autarkic bilateral oligopoly equilibrium will exist in $\mathcal{E}^{\bar{m}}$ and for all larger economies. In the appendix, we examine the sequence $\left\{\tilde{B}^{m}\right\}_{m=\bar{m}}^{\infty}$ and prove the remaining assertions in the following theorem.

Theorem 4.2 If Assumption A1 is satisfied, (6) holds and the preferences of all buyers are binormal, there is a non-autarkic equilibrium of $\mathcal{E}^{m}$ for all large enough $m$. Furthermore, as $m \rightarrow \infty$, strategic price in this equilibrium converges to the price $\tilde{p}$ on the equilibrium path of $\mathcal{G}^{-}$, each seller's output converges to their output on the same equilibrium path and the consumption choices of each buyer type approach those under price-taking behavior with price $\tilde{p}$.

This key result demonstrates that the behavior of sellers in the marketshare game can be seen as a good approximation to bilateral oligopoly with many buyers and that buyers approximately play the price-taking role assigned to them in the market share game. Since we also showed in the previous section that outcomes at the equilibrium of $\mathcal{G}^{0}$ and on the equilibrium path of $\mathcal{G}^{-}$are identical, we may conclude that $\mathcal{G}^{0}$ may also be viewed as an approximation to $\mathcal{E}^{m}$ when $m$ is large.

\section{Cournot and market share games}

In this section, we compare the outputs in Cournot and market share games and use the results to investigate the relationship between bilateral oligopoly with many buyers and Cournot competition. It will prove convenient to confine our attention to to the case in which demand is continuously differentiable where positive.

\subsection{Analyzing the Cournot game}

Consider the game $\mathcal{G}^{+}$, in which the auctioneer moves second. In any second-stage subgame in which aggregate output in the first stage is $X$, the auctioneer's best response is to choose $R=X p(X)$. In a subgame perfect equilibrium, the first-stage game becomes a Cournot game with payoffs given by (2) with $X p(X)$ in place of $R$. We will write $\mathcal{G}^{\mathrm{C}}$ for this Cournot game. It is well known (see, for instance, Example 2 in Novshek [12]) that 
the assumptions we have made so far are not sufficiently strong to ensure that a Cournot equilibrium exists. Rather than strengthen these conditions (and weaken our conclusions), we will maintain our assumptions and confine attention to those problems where a Cournot equilibrium does exist. Once again, we exploit the aggregative properties of the game.

The necessary first-order condition for $x_{i}$ to be a best response to $\mathbf{x}_{-i}$ in $\mathcal{G}^{\mathrm{C}}$ is

$$
\frac{\partial \pi_{i}}{\partial x_{i}}=p(X)+x_{i} p^{\prime}(X)-C_{i}^{\prime}\left(x_{i}\right) \leq 0,
$$

with equality if $x_{i}>0$. Re-writing these conditions in terms of market share $\sigma_{i}=x_{i} / X$ gives

$$
L^{\mathrm{C}}\left(\sigma_{i}, X\right)=\left[1-\frac{\sigma_{i}}{\eta(X)}\right] p(X) \leq C_{i}^{\prime}\left(\sigma_{i} X\right),
$$

with equality if $\sigma_{i}>0$, where $\eta(X)=-p(X) / X p^{\prime}(X)$ is the elasticity of demand.

Since $L^{\mathrm{C}}$ is strictly decreasing and $C_{i}^{\prime}\left(\sigma_{i} X\right)$ non-decreasing in $\sigma_{i}$, for all $X>0$, there is a unique $\sigma_{i}$ which satisfies these conditions if and only if $L^{\mathrm{C}}$ is not greater than $C_{i}^{\prime}$ at $\sigma_{i}=1$, that is, if

$$
\left[1-\frac{1}{\eta(X)}\right] p(X) \leq C_{i}^{\prime}(X) \text {. }
$$

If we let $\mathbb{X}_{i}$ denote the (non-empty) set of $X$ satisfying (10), there is a function $s_{i}^{\mathrm{C}}: \mathbb{X}_{i} \rightarrow[0,1]$, where, for any $X \in \mathbb{X}_{i}, s_{i}^{\mathrm{C}}(X)$ is the unique $\sigma_{i}$ satisfying (9). It follows that, if $\tilde{\mathbf{x}}^{\mathrm{C}}$ is a Cournot equilibrium and $\tilde{X}^{\mathrm{C}}=$ $\sum_{j \in I^{\mathrm{S}}} \tilde{x}_{j}^{\mathrm{C}}$, then $\tilde{X}^{\mathrm{C}} \in \bigcap_{j \in I^{\mathrm{S}}} \mathbb{X}_{j}$ and $\tilde{x}_{i}^{\mathrm{C}} / \tilde{X}^{\mathrm{C}}=s_{i}^{\mathrm{C}}\left(\tilde{X}^{\mathrm{C}}\right)$ for all $i \in I^{\mathrm{S}}$. Note that this implies

$$
\sum_{j \in I^{\mathrm{S}}} s_{j}^{\mathrm{C}}\left(\tilde{X}^{\mathrm{C}}\right)=1
$$

These facts will be used in the sequel to compare equilibria in the marketshare game with Cournot equilibria. Note, however, that $s_{i}^{\mathrm{C}}$ is not a share function, since, although necessary, the first-order conditions are not sufficient for best responses. So $\sum_{j \in I^{\mathrm{S}}} s_{j}^{\mathrm{C}}(X)=1$ does not imply that $X$ is a Cournot equilibrium level of output. Furthermore, without further restrictions on the demand function, $s_{i}^{\mathrm{C}}$ need not be strictly decreasing where positive. Nevertheless, by comparing $s_{i}^{\mathrm{C}}(X)$ and $s_{i}(X ; R)$ we can explore the relationship between $\mathcal{G}^{\mathrm{C}}$ and $\mathcal{G}(R)$, and by extension, between $\mathcal{G}^{+}$and $\mathcal{G}^{-}$.

\subsection{Unit elasticity}

It is an immediate consequence of the expressions for $L^{\mathrm{M}}$ and $L^{\mathrm{C}}$ (see (4) and (9) respectively) that, if $\eta(X)=1$ and $R=X p(X)$, then $L^{\mathrm{C}}\left(\sigma_{i}, X\right)=$ 
$L^{\mathrm{M}}\left(\sigma_{i}, X ; R\right)$ for all $\sigma_{i} \in[0,1]$. Consequently, if $\mathcal{G}^{\mathrm{C}}$ has an equilibrium with aggregate output $\tilde{X}^{\mathrm{C}}$, where $\eta\left(\tilde{X}^{\mathrm{C}}\right)=1$ and $R=\tilde{X}^{\mathrm{C}} p\left(\tilde{X}^{\mathrm{C}}\right)$, then $s_{i}\left(\tilde{X}^{\mathrm{C}} ; R\right)=s_{i}^{\mathrm{C}}\left(\tilde{X}^{\mathrm{C}}\right)$ and therefore

$$
\sum_{j \in I^{\mathrm{S}}} s_{j}\left(\tilde{X}^{\mathrm{C}} ; R\right)=\sum_{j \in I^{\mathrm{S}}} s_{j}^{\mathrm{C}}\left(\tilde{X}^{\mathrm{C}}\right)=1
$$

which implies that $\tilde{X}^{\mathrm{C}}$ is the equilibrium value of $X$ in $\mathcal{G}(R)$. Hence, $\tilde{X}^{\mathrm{C}}=$ $\mathcal{X}(R)$, where $R=\mathcal{X}(R) p(\mathcal{X}(R))$. It follows that $\mathcal{G}^{-}$has an equilibrium in which the auctioneer chooses $R=\tilde{X}^{\mathrm{C}} p\left(\tilde{X}^{\mathrm{C}}\right)$ in the first stage and seller $i$ chooses $s_{i}\left(\tilde{X}^{\mathrm{C}} ; R\right) \tilde{X}^{\mathrm{C}}=s_{i}^{\mathrm{C}}\left(\tilde{X}^{\mathrm{C}}\right) \tilde{X}^{\mathrm{C}}=\tilde{x}_{i}^{\mathrm{C}}$ on the equilibrium path. This proves the following result.

Proposition 5.1 Suppose Assumption A1 holds, demand is differentiable where positive and $\mathcal{G}^{+}$has an equilibrium at which elasticity of demand is unity. Then $\mathcal{G}^{-}$has a non-autarkic equilibrium at which outputs of each seller are equal on the equilibrium paths of $\mathcal{G}^{-}$and $\mathcal{G}^{+}$.

Note that it follows from the proposition that aggregate demand and therefore price agree on the equilibrium paths.

We may also conclude from the output equivalence of $\mathcal{G}^{-}$and $\mathcal{G}^{0}$, established in the previous section, that, under the suppositions of Proposition $5.1, \mathcal{G}^{0}$ has a non-autarkic equilibrium in which the outputs of all sellers are equal to those on the equilibrium path $\mathcal{G}^{+}$.

If all buyer types have Cobb-Douglas preferences, not only does demand have unit elasticity (and is strictly concave), but standard existence results imply that a Cournot equilibrium exists and is unique (see Szidarovsky and Yakowitz [17]). Moreover, Assumption A2 holds. This leads to the following corollary.

Corollary 5.2 Suppose Assumption A1 holds and all buyer types have Cobb-Douglas preferences, then $\mathcal{G}^{-}$and $\mathcal{G}^{+}$have subgame perfect equilibria and $\mathcal{G}^{0}$ has a non-autarkic Nash equilibrium in which equilibrium outputs are the same in all three games.

\subsection{A comparison of oligopoly models}

When elasticity differs from unity, we can use our share function constructions to order the outputs in the Cournot and market share games at the aggregate level. The following proposition summarizes the result, proved in the appendix. 
Proposition 5.3 Suppose Assumptions A1 and A2 hold, (6) is satisfied and competitive demand is differentiable where positive. If the aggregate output of sellers in the unique non-autarkic subgame perfect equilibrium of $\mathcal{G}^{-}$is denoted by $\tilde{X}$, and there is a Cournot equilibrium with aggregate output $\tilde{X}^{\mathrm{C}}$, then

$$
\eta\left(\tilde{X}^{\mathrm{C}}\right)>[<] 1 \Leftrightarrow \tilde{X}<[>] \tilde{X}^{\mathrm{C}} .
$$

In contrast to the case of unit elasticity, this result holds purely at the aggregate level. There are demand functions and sets of non-identical cost functions satisfying the suppositions of the proposition for which $\mathcal{G}^{\mathrm{C}}$ has an equilibrium with $\eta\left(\tilde{X}^{\mathrm{C}}\right)>1$, but in which there are individual sellers whose output is higher in $\mathcal{G}^{-}$than $\mathcal{G}^{+}$. This possibility arises because, even though $s_{i}(X ; R)<s_{i}^{\mathrm{C}}(X)$ when $R$ takes the equilibrium value in $\mathcal{G}^{-}$, the fact that $s_{i}$ is decreasing in $X$ prevents us from ruling out the possibility that $\tilde{x}_{i}=\tilde{X} s_{i}(\tilde{X} ; \tilde{R})>\tilde{X}^{\mathrm{C}} s_{i}\left(\tilde{X}^{\mathrm{C}}\right)=\tilde{x}_{i}^{\mathrm{C}}$.

The observations made in Subsection 3.3 show that comparisons between $\mathcal{G}^{+}$and $\mathcal{G}^{0}$ run the same way.

Corollary 5.4 Under the conditions of Proposition 5.3, (11) holds, where $\tilde{X}$ now denotes the aggregate output of sellers in the unique Nash equilibrium of $\mathcal{G}^{0}$.

To see the intuition behind Proposition 5.3 and Corollary 5.4, suppose there is an equilibrium in the market share game in which total revenue is $R$. When making supply decisions in this game, sellers consider only the effect of changes in their supply on their share of this total revenue, for they see its magnitude as fixed at the value $\tilde{R}$. In seeking their optimal choice, each seller equates the marginal benefit of extra supply to marginal cost (keeping the supply decisions of others fixed), the marginal benefit being the effect of a change in supply on their share of the revenue. If we were to impose the rules of the game $\mathcal{G}^{+}$at the equilibrium of $\mathcal{G}^{-}$, sellers would now consider the actions of the auctioneer who is determining the revenue $R$ at the second stage. As such, they must take into account that their supply decisions influence not only their share of the revenue but also the size of the total revenue to be shared amongst all sellers. When elasticity is unity, total revenue is fixed on the margin leading to identical outcomes in $\mathcal{G}^{+}$and $\mathcal{G}^{-}$. When elasticity is less than one, the direct marginal benefit of a small increase in supply is offset by a reduction in total revenue, leading to a lower total equilibrium output in $\mathcal{G}^{+}$than $\mathcal{G}^{-}$. Similarly, when elasticity exceeds one, an increase in supply increases total revenue and this reverses the ordering of total output. 


\subsection{Oligopoly with many buyers}

In this subsection, we combine the observation in Section 4, that quantity competition in bilateral oligopoly with many buyers can be approximated by the games $\mathcal{G}^{-}$(or $\mathcal{G}^{0}$ ), with the comparison of outcomes in $\mathcal{G}^{-}$and $\mathcal{G}^{+}$ conducted in the preceding subsections. This will allow us to show that the results in Examples 1 and 2 are typical rather than exceptional. We first apply Theorem 4.2 and Proposition 5.1 to deduce the following result.

Corollary 5.5 If, under the assumptions of Theorem 4.2, market demand is differentiable where positive and the Cournot game has an equilibrium at which elasticity of demand is one, then $\mathcal{E}^{m}$ has a unique non-autarkic equilibrium for all large enough $m$ in which the equilibrium outputs of all sellers approach those at the Cournot equilibrium.

A fortiori, this conclusion holds if cost functions satisfy Assumption A1 and all buyers have Cobb-Douglas preferences. The corollary suggests that Cournot oligopoly can then be viewed as an approximation of bilateral oligopoly with many buyers and bilateral oligopoly can provide a strategic foundation for Cournot competition under these circumstances.

When a Cournot equilibrium exists, but the equilibrium elasticity differs from unity, the best we can do is to use Theorem 4.2 and Proposition 5.3 to provide an ordering of aggregate outputs.

Corollary 5.6 Suppose the assumptions of Theorem 4.2 are satisfied, market demand is differentiable where positive and the Cournot game has an equilibrium with aggregate output $\tilde{X}^{\mathrm{C}}$ at which elasticity of demand is $\eta\left(\tilde{X}^{\mathrm{C}}\right)>$ $[<] 1$. Then there is a positive integer $M$ such that $\mathcal{E}^{m}$ has a unique nonautarkic equilibrium in which aggregate output $\tilde{X}^{m}$ satisfies $\tilde{X}^{m}<[>] \tilde{X}^{\mathrm{C}}$ for all $m \geq M$.

In this case, bilateral oligopoly does not provide a useful foundation for Cournot competition.

\section{Conclusion}

We have shown that equilibria in bilateral oligopoly with many buyers can be approximated by those in the market share game. Yet, there are inherent differences between equilibria in the market share game and Cournot equilibria unless the elasticity of demand at the latter is unity. This means Cournot competition is not generally a good approximation to bilateral oligopoly, even when the number of buyers is large. In markets with a competitive demand side, our results also imply that commitment to supply decisions before demand is realized is a crucial feature of Cournot competition. Absent this commitment, the game played is the market share game, and outcomes will generally be different. 


\section{A Appendix}

Proof of Proposition 3.3. For any $X>0$, the share function $s_{i}(X ; R)$ of seller $i$, has a finite limit as $R \rightarrow \infty$ and it is a straightforward consequence of the first-order conditions that this limit is unity. It follows that $S(X ; R)$ exceeds one for all large enough $R$ and therefore $\mathcal{X}(R)>X$, so $\mathcal{X}$ is unbounded.

The proof that dissipation is non-increasing exploits the fact that, for fixed $\rho>0$, the share function of seller $i$ is non-increasing in $R$ : if $R^{\prime}>R>$ 0 , then $s_{i}(\rho R ; R) \geq s_{i}\left(\rho R^{\prime} ; R^{\prime}\right)$. To see this, note that $\sigma_{i}=s_{i}(\rho R ; R)$ if and only if $L^{\mathrm{M}}\left(\sigma_{i}, \rho R ; R\right) \leq C_{i}^{\prime}\left(\sigma_{i} \rho R\right)$ with equality if $\sigma_{i}>0$, where, from (4), we have $L^{\mathrm{M}}\left(\sigma_{i}, \rho R ; R\right)=\left(1-\sigma_{i}\right) \rho$. For fixed $\sigma_{i} \in[0,1]$, an increase in $R$ leaves $L^{\mathrm{M}}$ unchanged and does not decrease $C_{i}^{\prime}\left(\sigma_{i} \rho R\right)$, by Assumption $\mathbf{A} 1$. Hence, $s_{i}(\rho R ; R)$ does not increase. (Note that this holds even if the share function falls to zero.)

Since

$$
\sum_{j \in I^{\mathrm{S}}} s_{j}(R \rho(R) ; R)=1
$$

an increase from $R$ to $R^{\prime}$ gives $\sum_{j \in I^{\mathrm{S}}} s_{j}\left(R^{\prime} \rho(R) ; R^{\prime}\right) \leq 1$. Since (12) holds if $R$ is replaced by $R^{\prime}$ and $s_{i}(X ; R)$ is non-increasing in $R$, we have $\rho\left(R^{\prime}\right) \leq \rho(R)$.

Proof of Theorem 3.4. The condition for $R>0$ to maximize the auctioneer's payoff can be re-written:

$$
\frac{R}{\mathcal{X}(R)}=p(\mathcal{X}(R))
$$

and we will show that the left hand side of (13) exceeds the right for all large enough $R$ and, subject to a certain extra condition, this inequality reverses for small $R$. Continuity will allow us to deduce the existence of positive $R$ satisfying (13).

Firstly, note that Proposition 3.3 implies that the left-hand side of (13) is either unbounded or has a positive limit as $R \rightarrow \infty$. The proposition also asserts that $\mathcal{X}(R) \rightarrow \infty$ as $R \rightarrow \infty$, which, under Assumption A2, implies that the right-hand side approaches or is equal to zero in this limit. These two observations mean that $R / \mathcal{X}(R)>p(\mathcal{X}(R))$ for all large enough $R$. To examine the limit $R \rightarrow 0$, we first observe that, since sellers can always choose no output and receive zero payoff, equilibrium payoffs must be non-negative. It follows that each seller's costs in equilibrium cannot exceed $R$ and therefore that each seller's equilibrium strategy approaches zero as $R \rightarrow 0$. We conclude that $\mathcal{X}(R) \rightarrow 0$ as $R \rightarrow 0$ and, if we write $p(0)$ for $\lim _{X \rightarrow 0} p(X)$ (and take $p(0)=+\infty$ if $p$ is unbounded), we see that the right-hand side of (13) approaches $p(0)$ as $R \rightarrow 0$. To calculate the 
limit of the left-hand side, we note that for a given $R>0$, the first-order conditions and (4) give, for any $i$,

$$
\left[1-s_{i}(\mathcal{X}(R) ; R)\right] \frac{R}{\mathcal{X}(R)} \leq C_{i}^{\prime}\left[s_{i}(\mathcal{X}(R) ; R) \mathcal{X}(R)\right],
$$

with equality if $s_{i}(\mathcal{X}(R) ; R)>0$. However, for all small enough $R>0$ every share function is positive, by Lemma 3.1, so equality holds for all $i$. Summing over $i$, dividing by $n-1$ and using $S(\mathcal{X}(R) ; R)=1$ yields

$$
\frac{R}{\mathcal{X}(R)}=\frac{1}{n-1} \sum_{j \in I^{\mathrm{S}}} C_{j}^{\prime}\left(\mathcal{X}(R) s_{j}(\mathcal{X}(R) ; R)\right) .
$$

Since $\mathcal{X}(R) \rightarrow 0$ as $R \rightarrow 0$, it follows from Lemma 3.1 that $R / \mathcal{X}(R) \rightarrow$ $\sum_{j \in I^{\mathrm{S}}} C_{j}^{\prime}(0) /(n-1)$. Provided this limit is less than $p(0)$ it follows that $R / \mathcal{X}(R)<p(\mathcal{X}(R))$ for all small enough $R$.

We have shown that, provided $p(0)$ is large enough, $R=\mathcal{X}(R) p(\mathcal{X}(R))$ for some $R>0$, by continuity. To see that this value is unique, note that Assumption A2 implies that $p(X)$ is strictly decreasing in $X$ and it follows from Proposition 3.3 (in particular the fact that $\mathcal{X}(R)$ is increasing in $R$ ) that the right-hand side of (13) is strictly decreasing in $R$. This proposition also implies that the left-hand side is non-decreasing, so (13) can have at most one solution.

Proof of Lemma 4.1. Consider a game of bilateral oligopoly in which the set of buyers is $I^{\mathrm{Bm}}$. A slight modification of the arguments in [6] show that, under the assumptions of the lemma, there is a share function $s_{i}^{m}(B ; p)$ for buyers of type $i \in \mathcal{I}^{\mathrm{B}}$ such that, in any non-autarkic equilibrium of this game with strategic price $p$, every buyer of type $i \in \mathcal{I}^{\mathrm{B}}$ bids $b_{i}^{m}=B^{m} s_{i}^{m}\left(B^{m} ; p\right)$, where the aggregate offer $B^{m}$ satisfies

$$
\sum_{j \in I^{\mathrm{B} m}} \frac{s_{j}^{m}\left(B^{m} ; p\right)}{m}=\sum_{j \in \mathcal{I}^{\mathrm{B}}} s_{j}^{m}\left(B^{m} ; p\right)=1 .
$$

Furthermore, if $\partial_{i}\left(y_{1}, y_{2}\right)$ denotes the marginal rate of substitution with $y_{1}$ units of the good and $y_{2}$ units of money and if $\partial_{i}\left(0, e_{i}\right) \leq p$, then $s_{i}^{m}(B ; p)=$ 0 for all $B>0$, whereas, if $\partial_{i}\left(0, e_{i}\right)>p$, then $s_{i}^{m}(B ; p)$ is the unique $\sigma \in[0,1]$ satisfying

$$
\partial_{i}\left(\frac{\sigma B}{p}, e_{i}-\sigma B\right)=\left(1-\frac{\sigma}{m}\right)^{-1} p .
$$

Since $B^{m} \in\left[0, \sum_{j \in \mathcal{I}^{\mathrm{B}}} e_{j}\right]$ and $s_{i}^{m}\left(B^{m} ; p\right) \in[0,1]$ for all $m$, there is a subsequence on which $B^{m} \longrightarrow B$ and $s_{i}^{m}\left(B^{m} ; p\right)$ converges to $\sigma_{i}(p)$ satisfying $\sum_{j \in \mathcal{I}^{\mathrm{B}}} \sigma_{j}(p)$ as $m \longrightarrow \infty$. Taking limits in (14), using the continuity 
of $\partial_{i}$ shows that $\sigma_{i}(p)=0$ if $\partial_{i}\left(0, e_{i}\right) \leq p$ and satisfies

$$
\partial_{i}\left(\frac{B \sigma_{i}(p)}{p}, e_{i}-B \sigma_{i}(p)\right)=p
$$

otherwise. Since share functions sum to one, $\sum_{j \in \mathcal{I}^{\mathrm{B}}} \sigma_{j}(p)=1$. Together with (15), this uniquely defines $B$ and $\sigma_{i}(p)$ for $i \in \mathcal{I}^{\mathrm{B}}$, which in turn shows these are the limits of the sequences $\left\{B^{m}\right\}$ and $\left\{s_{i}^{m}\left(B^{m} ; p\right)\right\}$ respectively. It follows from (15) that, if $x_{i}$ denotes the competitive demand of buyers of type $i$ at price $p$, we have $B \sigma_{i}(p)=p x_{i}$. Writing $\mathcal{D}^{m}(p)=B^{m} / p$ for the strategic demand of buyers at price $p$, we have

$$
\mathcal{D}^{m}(p)=\frac{B^{m}}{p} \sum_{j \in \mathcal{I}^{\mathrm{B}}} s_{j}^{m}\left(B^{m} ; p\right) \longrightarrow \frac{B}{p} \sum_{j \in \mathcal{I}^{\mathrm{B}}} \sigma_{j}(p)=\mathcal{D}(p),
$$

where $\mathcal{D}(p)=\sum_{j \in \mathcal{I}^{\mathrm{B}}} x_{j}$ is the competitive demand at price $p$. Under binormality and Assumptions $\mathbf{A} \mathbf{1}$ and $\mathbf{A 2}, \mathcal{D}^{m}$ and $\mathcal{D}$ are continuous and strictly decreasing, so we can invert these functions to deduce pointwise convergence of inverse demand. We can extend this to uniform convergence on compact sets by showing that convergence is monotone and applying Dini's theorem.

We can prove that convergence is monotone by first showing that $s_{i}^{m}(B ; p)$ is non-decreasing in $m$. Note that a rise in $\sigma$ increases $\sigma B / p$ and decreases $e_{i}-\sigma B$ and binormality implies that the left hand side of (14) is decreasing in $\sigma$, whereas the right hand side of (14) is increasing in $\sigma$. Furthermore, a rise in $m$ decreases the right hand side of (14) and therefore the solution to (14) increases. Hence, $s_{i}^{m}(B ; p)<s_{i}^{m+1}(B ; p)$ if $\partial_{i}\left(0, e_{i}\right)>p$ and $s_{i}^{m}(B ; p)=s_{i}^{m+1}(B ; p)=0$ if $\partial_{i}\left(0, e_{i}\right) \leq p$. It follows that

$$
\sum_{j \in \mathcal{I}^{\mathrm{B}}} s_{j}^{m+1}\left(B^{m} ; p\right) \geq \sum_{j \in \mathcal{I}^{\mathrm{B}}} s_{j}^{m}\left(B^{m} ; p\right)=1 .
$$

Since it follows from the argument in $[6]$ that $\sum_{j \in \mathcal{I}^{\mathrm{B}}} s_{j}^{m+1}(B ; p)$ is decreasing in $B$ where positive and is equal to 1 at $B=B^{m+1}$, we deduce that $B^{m+1} \geq$ $B^{m}$ and therefore $\mathcal{D}^{m+1}(p) \geq \mathcal{D}^{m}(p)$. By inverting these functions we can conclude that inverse demand functions are also ordered by $m$.

Proof of Theorem 4.2. The sequence $\left\{\tilde{B}^{m}\right\}_{m=\bar{m}}^{\infty}$ satisfies $0 \leq \tilde{B}^{m} \leq$ $\sum_{j \in \mathcal{I}^{\mathrm{B}}} e_{j}$ for all $m \geq \bar{m}$, which means that it will have at least one accumulation point, which we write $\tilde{B}$. Continuity of $\mathcal{X}$ (Proposition 3.3) implies that $\mathcal{X}\left(\tilde{B}^{m}\right) \longrightarrow \mathcal{X}(\tilde{B})$ as $m \longrightarrow \infty$. Furthermore, the sequence $\left\{\mathcal{X}\left(\tilde{B}^{m}\right)\right\}_{m=\bar{m}}^{\infty}$ is contained in a compact set, so we may exploit uniformity of convergence from Lemma 4.1 to deduce that $p^{\mathrm{B} m}\left(\mathcal{X}\left(\tilde{B}^{m}\right)\right) \longrightarrow$ 
$p(\mathcal{X}(\tilde{B}))$. Applying this to $(7)$ shows that $\tilde{B}=f(\mathcal{X}(\tilde{B}))$, where $f$ is the revenue function $f(X)=X p(X)$. It follows that $\tilde{B}$ is the aggregate revenue in a subgame perfect equilibrium of $\mathcal{G}^{-}$. We have noted that the assumptions of the theorem imply that $p$ satisfies Assumption A2, which means we can apply Theorem 3.4 to deduce that $\tilde{B}$ is unique. This shows that $\tilde{B}^{m} \longrightarrow \tilde{B}$ as $m \longrightarrow \infty$ and that $\tilde{B}$ is the equilibrium aggregate revenue in the market share game.

Continuity of $\mathcal{X}$ allows us to deduce that $\tilde{X}^{m}$, the aggregate output in this sequence of equilibria, converges to $\tilde{X}$, the aggregate output in the equilibrium of the market share game: $\tilde{X}^{m}=\mathcal{X}\left(\tilde{B}^{m}\right) \rightarrow \mathcal{X}(\tilde{B})=\tilde{X}$ as $m \rightarrow \infty$. This also implies that strategic price $\tilde{p}^{m}$ converges to $\tilde{p}=p(\tilde{X})$ in the same limit.

Examining the payoffs of sellers for a given aggregate bid $B$ in (1) shows that sellers in $\mathcal{E}^{m}$ can be viewed as competing in a contest with prize $\tilde{B}^{m}$. We can use the analysis in Subsection 3.1 to deduce that the equilibrium output of seller $i$ is $\tilde{x}_{i}^{m}=\tilde{X}^{m} s_{i}\left(\tilde{X}^{m} ; \tilde{B}^{m}\right)$, where $s_{i}$ is the share function of seller $i$. Continuity of $s_{i}$ (Lemma 3.1) implies that $\tilde{x}_{i}^{m} \rightarrow \tilde{X} s_{i}(\tilde{X} ; \tilde{B})=\tilde{x}_{i}$, the output of seller $i$ on the equilibrium path of $\mathcal{G}^{-}$.

If buyers of type $i$ bid $\tilde{b}_{i}^{m}$ in the non-autarkic equilibrium of $\mathcal{E}^{m}$, then

$$
u_{i}\left(\frac{\tilde{b}_{i}^{m} \tilde{X}^{m}}{\tilde{B}^{m}}, e_{i}-\tilde{b}_{i}^{m}\right) \geq u_{i}\left(\frac{m b_{i} \tilde{X}^{m}}{m \tilde{B}^{m}-\tilde{b}_{i}^{m}+b_{i}}, e_{i}-b_{i}\right)
$$

for all $b_{i} \in\left[0, e_{i}\right]$. Since $\tilde{b}_{i}^{m} \in\left[0, e_{i}\right]$ for all $m \geq \bar{m}$, the sequence $\left\{\tilde{b}_{i}^{m}\right\}_{m=\bar{m}}^{\infty}$ has a subsequence convergent to $\tilde{b}_{i}$, say. Taking the limit in this inequality gives

$$
u_{i}\left(\frac{\tilde{b}_{i}}{\tilde{p}}, e_{i}-\tilde{b}_{i}\right) \geq u_{i}\left(\frac{b_{i}}{\tilde{p}}, e_{i}-b_{i}\right),
$$

for all $b_{i} \in\left[0, e_{i}\right]$. This says that $\tilde{b}_{i} / \tilde{p}$ is the expenditure on the consumption good of a price-taking consumer with utility function $u_{i}$ and facing price $\tilde{p}$. Since this makes $\tilde{b}_{i}$ unique, we conclude that $\tilde{b}_{i}^{m} \rightarrow \tilde{b}_{i}$ as $m \rightarrow \infty$.

We now turn to the proof of Proposition 5.3. The proof uses the following lemma.

Lemma A.1 Suppose that Assumption A1 holds, competitive demand is differentiable where positive and there is an equilibrium of $\mathcal{G}^{\mathrm{C}}$ with aggregate supply $\tilde{X}^{\mathrm{C}}$, then

$$
R \leq[\geq] f\left(\tilde{X}^{\mathrm{C}}\right) \text { and } \eta\left(\tilde{X}^{\mathrm{C}}\right)>[<] 1 \Rightarrow \mathcal{X}(R)<[>] \tilde{X}^{\mathrm{C}},
$$

where $f(X)=X p(X)$. 
Proof. Suppose $R, X>0$ satisfy $R \leq X p(X)$ and $\eta(X)>1$. It follows from (4) and (9) that $L\left(\sigma_{i}, X ; R\right) \leq L^{\mathrm{C}}\left(\sigma_{i}, X\right)$ with strict inequality unless $\sigma_{i}=0$ and $R=X p(X)$. Since the right-hand sides of the first-order conditions (marginal costs) are identical in both games, we can deduce that $s_{i}(X ; R) \leq s_{i}^{\mathrm{C}}(X)$ for all $X \in \mathbb{X}$ and the inequality is strict if $s_{i}^{\mathrm{C}}(X)>0$ or $R<X p(X)$. This is illustrated in Figure 2, which displays the construction of these functions in the games $\mathcal{G}(R)$ and $\mathcal{G}^{\mathrm{C}}$.

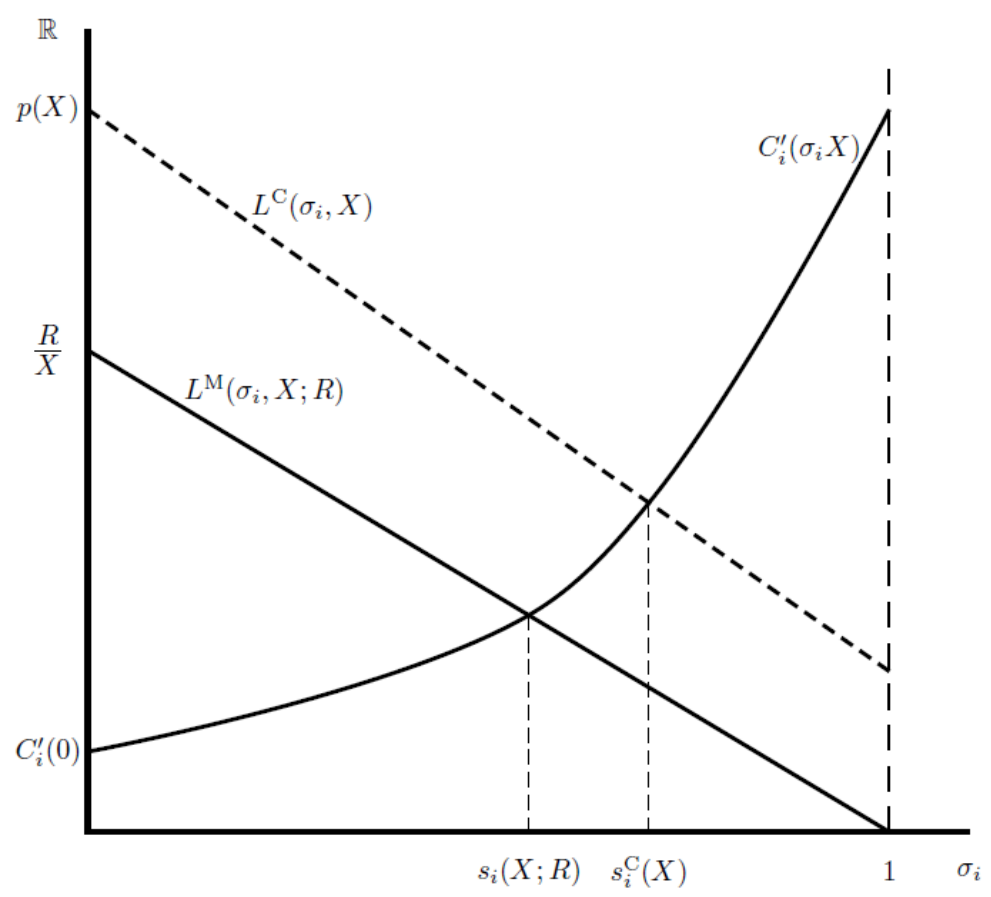

Figure 2: Comparing share functions in Cournot competition (dashed) and the market-share game (solid) when $R \leq X p(X)$ and $\eta(X)>1$.

We can apply this deduction to a comparison of equilibria in $\mathcal{G}^{\mathrm{C}}$ and $\mathcal{G}(R)$, so suppose $\mathcal{G}^{\mathrm{C}}$ has an equilibrium with aggregate supply $\tilde{X}^{\mathrm{C}}$. If $R \leq \tilde{X}^{\mathrm{C}} p\left(\tilde{X}^{\mathrm{C}}\right)$ and $\eta\left(\tilde{X}^{\mathrm{C}}\right)>1$, we can sum our result over all sellers to obtain

$$
\sum_{j \in I^{\mathrm{S}}} s_{j}\left(\tilde{X}^{\mathrm{C}} ; R\right)<\sum_{j \in I^{\mathrm{S}}} s_{j}^{\mathrm{C}}\left(\tilde{X}^{\mathrm{C}}\right)=1 .
$$

Since $\sum_{j \in I^{\mathrm{S}}} s_{j}(X ; R)$ is continuous and strictly decreasing in $X$ where positive, and $\sum_{j \in I^{\mathrm{S}}} s_{j}(\mathcal{X}(R) ; R)=1$, we can deduce that $\mathcal{X}(R)<\tilde{X}^{\mathrm{C}}$.

The proof when the inequalities are reversed is similar.

Proof of Proposition 5.3. We consider the case in which the assumptions 
of the proposition hold and $\eta\left(\tilde{X}^{\mathrm{C}}\right)>1$. Applying Lemma A.1 with $R=$ $f\left(\tilde{X}^{\mathrm{C}}\right)$ allows us to deduce that $\mathcal{X}\left(f\left(\tilde{X}^{\mathrm{C}}\right)\right)<\tilde{X}^{\mathrm{C}}$ and therefore

$$
\frac{\mathcal{X}\left(f\left(\tilde{X}^{\mathrm{C}}\right)\right)}{f\left(\tilde{X}^{\mathrm{C}}\right)}<\frac{\tilde{X}^{\mathrm{C}}}{f\left(\tilde{X}^{\mathrm{C}}\right)}=\frac{1}{p\left(\tilde{X}^{\mathrm{C}}\right)} .
$$

Now, using $\tilde{X}$ to denote the aggregate output in the non-null equilibrium of $\mathcal{G}^{-}$and putting $\tilde{R}=f(\tilde{X})$, we have

$$
\frac{1}{p(\mathcal{X}(\tilde{R}))}=\frac{\mathcal{X}(\tilde{R})}{f(\mathcal{X}(\tilde{R}))}=\frac{\mathcal{X}(\tilde{R})}{\tilde{R}} .
$$

We will prove that $\tilde{X}<\tilde{X}^{\mathrm{C}}$ by contradiction, so note that if we had $\tilde{X}=$ $\mathcal{X}(\tilde{R}) \geq \tilde{X}^{\mathrm{C}}$, the fact that demand is decreasing by Assumption A2 would imply that the right-hand side of (17) would not exceed the left-hand side of (18). But this implies

$$
\frac{\mathcal{X}\left(f\left(\tilde{X}^{\mathrm{C}}\right)\right)}{f\left(\tilde{X}^{\mathrm{C}}\right)}<\frac{\mathcal{X}(\tilde{R})}{\tilde{R}}
$$

and the fact that $\mathcal{X}(R) / R$ is non-increasing (Proposition 3.3) implies $f\left(\tilde{X}^{\mathrm{C}}\right)>$ $\tilde{R}$. A further application of Lemma A.1 would yield $\mathcal{X}(\tilde{R})<\tilde{X}^{\mathrm{C}}$, the claimed contradiction. A similar argument allows us to deduce that $\eta\left(\tilde{X}^{\mathrm{C}}\right)<$ 1 implies $\tilde{X}=\mathcal{X}(\tilde{R})>\tilde{X}^{\mathrm{C}}$.

\section{References}

[1] Busetto, F., G. Codognato and S. Ghosal; 2008; "Cournot-Walras equilibrium as a subgame perfect equilibrium"; International journal of game theory; 37 , pp 371-86

[2] Codognato, G.; 1995; "Cournot-Walras and Cournot Equilibria in Mixed Markets: A Comparison"; Economic Theory; 5,pp 361-70.

[3] Cornes, R. and R. Hartley; 2005; "Asymmetric contests with general technologies"; Economic Theory; 26(4) pp 923-46.

[4] Cornes, R. and R. Hartley; 2005; "The geometry of aggregative games"; University of Manchester economics discussion paper 0514. 
[5] Cournot, A.; 1838; Researches into the Mathematical Principles of the Theory of Wealth; English translation by Bacon, N.; 1897; Macmillan, New York.

[6] Dickson, A. and R. Hartley; 2008; "The Strategic Marshallian Cross"; Games and Economic Behavior; 64(2) pp 514-32.

[7] Dubey, P.; 1994; "Strategic market games: a survey of some results"; in: J-F Mertens, S. Sorin (Eds.), Game-theoretic methods in general equilibrium analysis, Kluwer, Amsterdam, pp209-24.

[8] Gabszewicz, J. J. and P. Michel; 1997; "Oligopoly equilibrium in exchange economies"; in: B. C. Eaton and R. G. Harris (Eds.), Trade, technology and economics: Essays in honor of Richard G. Lipsey; Elgar, Cheltenham, UK, pp 217-240.

[9] Gabszewicz, J. J. and J.-P. Vial; 1972; "Oligopoly "a la Cournot" in a general equilibrium analysis"; Journal of Economic Theory; 4 pp 381400 .

[10] Giraud, G.; (2003) "Strategic market games: an introduction"; Journal of Mathematical Economics; 39 pp 355-75.

[11] Mas-Colell, A.; 1982; "The Cournotian Foundations of Walrasian Equilibrium Theory: An Exposition of Recent Theory"; in Advances in Economic Theory, Hildenbrand, W (ed.); Cambridge University Press; pp 183-224.

[12] Novshek, W.; 1985; "On the Existence of Cournot Equilibrium"; Review of Economic Studies; 52, pp 85-98.

[13] Selten, R.; 1970; Preispolitik der Mehrproduktenunternehmung in der Statischen Theorie, Springer-Verlag.

[14] Shapley, L. and M. Shubik; 1977; "Trade Using One Commodity as a Means of Payment"; Journal of Political Economy; 85(5), pp 937-68.

[15] Stein, W.; 2002; "Asymmetric rent-seeking with more than two contestants"; Public Choice; 113, pp 325-36.

[16] Szidarovsky, F. and K. Okuguchi; 1997; "On the existence and uniqueness of pure Nash equilibrium in rent-seeking games"; Games and Economic Behavior; 18 pp 135-40.

[17] Szidarovsky, F. and S. Yakowitz; 1977; "A new proof of the existence and uniqueness of the Cournot equilibrium"; International Economic Review; 18, pp 787-9.

[18] Vives, X.; 1999; Oligopoly pricing: old ideas and new tools; MIT press. 